\title{
Feline Foamy Virus Adversely Affects Feline Mesenchymal Stem Cell Culture and Expansion: Implications for Animal Model Development
}

\author{
Boaz Arzi, ${ }^{1, *}$ Amir Kol, ${ }^{2, *}$ Brian Murphy, ${ }^{2}$ Naomi J. Walker, ${ }^{2}$ Joshua A. Wood, ${ }^{2}$ \\ Kaitlin Clark, ${ }^{2}$ Frank J.M. Verstraete, ${ }^{1}$ and Dori L. Borjesson ${ }^{2}$
}

\begin{abstract}
Mesenchymal stem cells (MSCs) are a promising therapeutic option for various immune-mediated and inflammatory disorders due to their potent immunomodulatory and trophic properties. Naturally occurring diseases in large animal species may serve as surrogate animal models of human disease, as they may better reflect the complex genetic, environmental, and physiologic variation present in outbred populations. We work with naturally occurring diseases in large animal species to better understand how MSCs work and to facilitate optimal translation of MSC-based therapies. We are investigating the use of MSC therapy for a chronic oral inflammatory disease in cats. During our efforts to expand fat-derived feline MSCs (fMSCs), we observed that $\sim 50 \%$ of the cell lines developed giant foamy multinucleated cells in later passages. These morphologic alterations were associated with proliferation arrest. We hypothesized that the cytopathic effects were caused by infection with a retrovirus, feline foamy virus (FFV). Using transmission electron microscopy, polymerase chain reaction, and in vitro assays, we determined that syncytial cell formation and proliferation arrest in fMSCs were caused by FFV strains that were highly homologous to previously reported FFV strains. We determined that the antiretroviral drug, tenofovir, may be used to support ex vivo expansion and salvage of FFV-infected fMSC lines. MSC lines derived from specific pathogen-free cats do not appear to be infected with FFV and may be a source of allogeneic fMSCs for clinical application. FFV infection of fMSC lines may hinder large-scale expansion of autologous MSC for therapeutic use in feline patients.
\end{abstract}

\section{Introduction}

A DIPOSE-DERIVED MESENCHYMAL stem cells (MSCs) have been isolated and characterized from several species, including domestic cats [1-4]. MSCs have a profound regenerative ability attributed, in part, to their ability to modulate both the innate and adaptive immune systems [5-7]. Although the mechanisms of immunomodulation remain partially elusive, MSCs inhibit T-cell proliferation, alter B-cell function, downregulate major histocompatibility complex (MHC) II, and inhibit dendritic cell maturation and differentiation [6,8-10]. Because of these functions, MSCs are currently in phase I-III human clinical trials for immunemediated diseases, including inflammatory alimentary tract diseases with promising results [11-14].

Animal models play an important role in preclinical safety and efficacy studies. Although experimentally induced ani- mal models of disease are important, they typically lack the complex genetic and environmental interactions that accompany large animal models of naturally occurring disease [15-17]. Our research group is focused on developing models of naturally occurring disease in large animal species that may complement experimentally induced animal models of human disease. Specifically, we focus on disease models that may serve as a platform to investigate MSCbased novel regenerative medicine therapies. For example, feline chronic gingivostomatitis (FCGS) is a common, chronic immune-mediated oral mucosal disease in cats that is the result of a dysregulated and aberrant immune response. FCGS has an incompletely understood etiopathogenesis [18-20]. The ability of MSCs to inhibit T-cell proliferation and modulate $\mathrm{T}$ - and $\mathrm{B}$-cell function suggests that FCGS may be an ideal lesion for MSC-based regenerative medicine therapy.

Departments of ${ }^{1}$ Surgical and Radiological Sciences and ${ }^{2}$ Microbiology and Immunology, School of Veterinary Medicine, University of California, Davis, Davis, California.

*These authors have contributed equally and are first co-authors. 
To expand a large number of MSCs for characterization and subsequent clinical administration, reproducible and reliable culture conditions must be established. In this study, we report the growth kinetics and surface phenotype of adipose-derived feline MSCs (fMSCs) obtained from specific pathogen-free (SPF) cats as well as from non-SPF donor animals. Fifty-five percent of cell lines obtained from client-owned cats contained large syncytial cells evident at passages 3-5. These cell lines failed to proliferate. We hypothesized that these effects were secondary to infection and active replication of a retrovirus, feline foamy virus (FFV). In this article, we demonstrate that FFV infection in fMSC lines poses a fundamental problem for long-term culture that may complicate the clinical application of autologous MSCs in cats. We further investigate options for bypassing the adverse effects of FFV on fMSC culture and clinical application.

\section{Materials and Methods}

\section{Tissue samples}

The studies were conducted according to a protocol approved by the Institutional Animal Care and Use Committee, and the Clinical Trials Review Board, University of California, Davis (UCD). Under general anesthesia, subcutaneous abdominal fat samples were obtained from three SPF cats and from nine client-owned cats. All owners had signed an informed consent form. All cats were free of feline immune deficiency virus and feline leukemia virus infection.

\section{fMSC isolation and expansion}

MSCs were isolated from fat and cultured exactly as previously described for canine MSCs [21-23]. MSCs were cultured in low-glucose Dulbecco's modified Eagle's medium (Mediatech), 10\% fetal bovine serum (HyClone, Inc.), and $1 \%$ penicillin/streptomycin (Thermo Fisher Scientific) in tissue culture flasks (Nunc) and incubated at $37^{\circ} \mathrm{C}, 5 \% \mathrm{CO}_{2}$. Cells were passaged once they reached $\sim 70 \%$ confluency.

\section{fMSC growth kinetics}

Five FFV-free fMSC lines were plated into two T25 flasks at 5,000 cells $/ \mathrm{cm}^{2}$. Cells were counted using a hemocytometer at each passage to determine growth kinetics (population doubling), to determine viability through trypan blue exclusion dye (Life Technologies), and to evaluate morphology. Population doubling times were calculated, as previously described [24]. Cultures were terminated when they stopped proliferating.

\section{fMSC phenotype}

Surface protein expression on fMSC lines (five FFVinfected and three FFV-free cell lines) was determined using flow cytometry. All antibodies were purchased from the Leukocyte Antigen Biology Laboratory, UCD, unless otherwise indicated. Antibodies included MHC II (42.3), CD18 (FE3.9F2), CD90 (CA1.4G8), CD44 (IM7; BioLegend), and CD105 (SN6; eBioscience). For unconjugated antibodies, a mouse IgG-phycoerythrin (PE) antibody (Jackson ImmunoResearch Labs) was used for secondary labeling. Canine CD8 $\alpha$ (CA9.JD3), rat immunoglobulin G-allophycocyanin
(IgG-APC) (eBR2a; eBioscience), and mouse IgG-APC (MCA928; AbD Serotec) were used as isotype controls. Samples were run on a flow cytometer (Cytomics FC500). Flow cytometry data were analyzed using FlowJo flow cytometry software (Tree Star, Inc.).

\section{Cytomorphology of FFV-infected fMSCs}

For cytologic examination, two fMSC lines were plated $\left(8 \times 10^{4}\right.$ per chamber) on multichamber glass slides (Nalge Nunc International) and were cultured for 3-5 days. Slides were stained with Hema $3^{\circledR}$ (Thermo Fisher Scientific) and evaluated with bright-field microscopy or they were stained with 4',6-diamidino-2-phenylindole (DAPI; Thermo Fisher Scientific) and Alexa Fluor 488 Phalloidin (Thermo Fisher Scientific) as per the manufacturer's instructions and examined with a fluorescent microscope (Olympus BX161; Olympus, Inc.). Digital images were captured with a digital camera (Penguin 600CL; Pixera Corporation) and a compatible computer software program (View Finder 3.0.1; Pixera Corporation).

\section{Transmission electron microscopy}

A single fMSC pellet was immersed in modified $(50 \%$ strength) Karnovsky's fixative, postfixed in 1\% osmium tetroxide, rinsed in $0.1 \mathrm{M}$ sodium cacodylate, dehydrated through a series of graded ethanol, transitioned through propylene oxide, and infiltrated and embedded in Eponate 12 epoxy formulation (Eponate-12 epoxy resin; Ted Pella, Inc.). Thick sections were stained with toluidine blue, and selected ultrathin sections were examined using a Zeiss 906E transmission electron microscope (Carl Zeiss SMT).

\section{FFV polymerase chain reaction and DNA sequencing}

To detect FFV-derived nucleic acids, a specific primer set was designed (forward primer: 5'-GAGATTGAGACTCTC CAGCTTGGGTAAGA, reverse primer: 5'-GCTCTAACA CTTTCAAATAGAAGAACACTTTTCCCC, product size: $206 \mathrm{bp}$ ) using an online computer software (Integrated DNA Technologies) designed to amplify the R and U5 segments of the viral long terminal repeat. Genomic DNA (gDNA) and RNA were extracted from cultured cells using an AllPrep DNA/RNA mini kit (Qiagen) as per the manufacturer's instructions. For the purpose of RNA extraction, gDNA digestion was performed using an RNase-Free DNase Set (Qiagen) as per the manufacturer's instructions. The cDNA was synthesized with the First-Strand cDNA Synthesis kit (OriGene) as per the manufacturer's instructions. Reverse transcription negative (RT-) controls were generated by omitting the reverse transcriptase from the mastermix used for cDNA synthesis. A polymerase chain reaction (PCR) assay was optimized (30 cycles, annealing temperature of $57^{\circ} \mathrm{C}$ for $30 \mathrm{~s}$ ) for both gDNA and reverse-transcribed cDNA samples. PCR products were concentrated and purified (Amicon Ultra kit; Millipore) as per the manufacturer's instructions and sequenced by a local vendor (Davis Sequencing). Resulting R/U5 sequences were aligned with FFV sequences available in the GenBank database; a phylogenetic dendrogram was created using the same set of sequences (Vector NTI; Invitrogen). 


\section{FFV western blot}

Ten and $20 \mu \mathrm{g}$ of FFV-infected MSCs lysate and FFV-free MSCs lysate were loaded into a 4\%-20\% SDS Precast Gels (Expedeon) and separated by electrophoresis. Proteins were transferred to polyvinylidene difluoride membranes overnight and then probed with cat sera at 1:2,000 dilution factor overnight. Membranes were washed, followed by $1 \mathrm{~h}$ incubation with horseradish peroxidase-conjugated rabbit anti-cat IgG antibody, and finally incubated for $1 \mathrm{~min}$ with Pierce ECL Western Blotting Substrate (Thermo Scientific). Digital images were obtained using a FluorChem E Imaging System (ProteinSimple).

\section{FFV cross infection}

Media from a FFV-infected fMSC culture (confirmed both by microscopic examination and by PCR) were aspirated and centrifuged (300 $\mathrm{g}$ for $5 \mathrm{~min}$ ); the supernatant was re-aspirated and centrifuged for a second time (2,500 $g$ for 5 min). FFV-negative fMSCs (a single cell line) were plated in duplicates into a six-well plate (Corning) and serial dilutions of media from a FFV-infected culture $(1: 2.5,1: 5$, $1: 10,1: 20)$ were added along with a control well with no FFV media. Cells were cultured for 5-7 days after which the cells were interrogated by PCR and morphologic analyses, as previously described.

\section{Tenofovir treatment}

A $100 \mathrm{mM}$ solution of tenofovir disoproxil fumarate (Selleckchem) was solvated in tissue culture grade dimethyl sulfoxide (Sigma). This stock was then added to culture media to create a $100 \mu \mathrm{M}$ stock that was filter sterilized. This stock was further diluted in culture media to create a range of tenofovir concentrations $(100,50,10,5,1$, and $0 \mu \mathrm{M})$.

\section{Results}

\section{FFV-free fMSCs display blunted proliferation ex vivo but have similar surface phenotype compared with MSCs from other species}

Growth kinetics and cell surface phenotype were determined on FFV-free fMSCs to better characterize these cells and optimize their culture conditions (Fig. 1). For fMSCs at passages $1-4$, doubling times were consistently $\sim 48 \mathrm{~h}$ (Fig. 1A). After passage 4, doubling times were markedly increased and inconsistent between different cell lines. Three of the five fMSC lines had a doubling time of $\sim 3$ days at passage 5 , and two fMSC lines had doubling times $>7$ days. This phenomenon was exacerbated with further passages, and only $1 / 5$ cell lines was still proliferative at passage 8 .

FFV-free fat-derived fMSCs had the expected MSC surface phenotype. fMSCs were uniformly negative for CD18 and MHC II and were mostly positive for CD105, CD44, and CD90 (Fig. 1B). Interestingly, FFV-infected MSCs had unaltered cell surface phenotype (Fig. 1B).

\section{MSCs from non-SPF cats often form multinucleated giant cells and undergo proliferation arrest}

MSCs from SPF cats have typical MSC morphology (elongate spindle-shaped cells, Fig. 2A-C). Five of the nine MSC lines that were cultured from client-owned cats formed giant
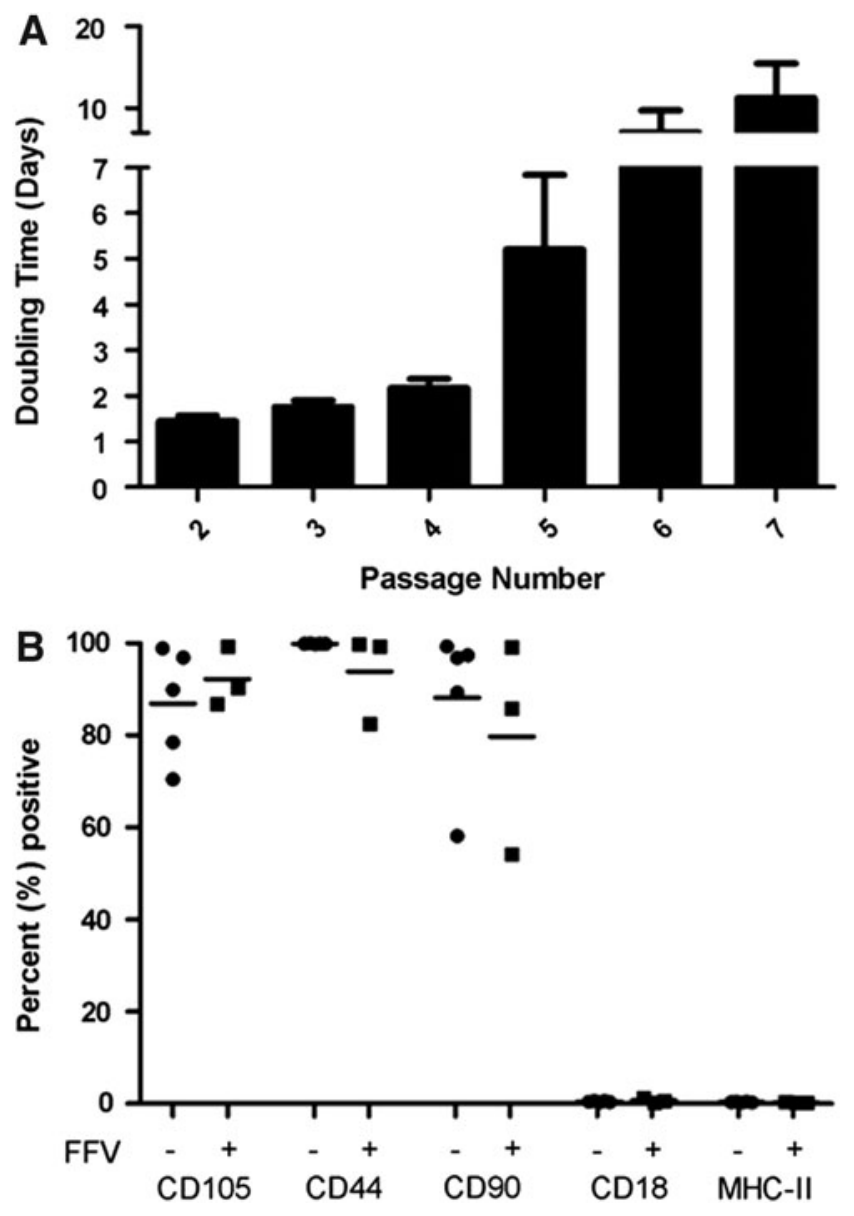

FIG. 1. fMSCs have typical cell surface phenotype and an attenuated proliferative capacity. Five FFV-free fMSC lines were used for cells surface phenotype and growth kinetics determination studies. Growth kinetics was determined by plating cells at 5,000 cells $/ \mathrm{cm}^{2}$ followed by culturing until $\sim 80 \%$ confluency was reached. Cells were then harvested, counted, replated, and doubling times were calculated. During the first three passages, doubling times were $<2$ days with low variability (A). As of the fourth passage, doubling times have started to gradually increase with greater variability between the different cell lines. Only one cell line remained proliferative at passage 8. Cell surface phenotype of FFV-free MSCs $(\bullet)$ indicated that cells were all negative for the hematopoietic markers (ie, CD18 and MHC II) (B). CD44 was highly expressed in all cell lines $(99.8 \% \pm 0.06 \%$; mean \pm standard deviation). CD105 and CD90 were primarily positively expressed $(86.9 \% \pm 12.2 \%$ and $88.2 \% \pm 17.2 \%$, respectively) (B). Three FFV positive MSC lines were further immunophenotyped ( $\mathbf{\square})$. No significant changes were noted in the FFV-infected cells (B). FFV, feline foamy virus; fMSCs, feline MSCs; MHC, major histocompatibility complex; MSCs, mesenchymal stem cells.

multinucleated syncytial cells between passages 3-5 (Fig. 2DF). Cells with this morphology underwent proliferation arrest.

\section{Morphologic, immunophenotypic, and proliferative alterations of $\mathrm{FMSCS}$ are associated with FFV infection}

We hypothesized that FFV was the cause of the morphologic and proliferative cell alterations given the high 

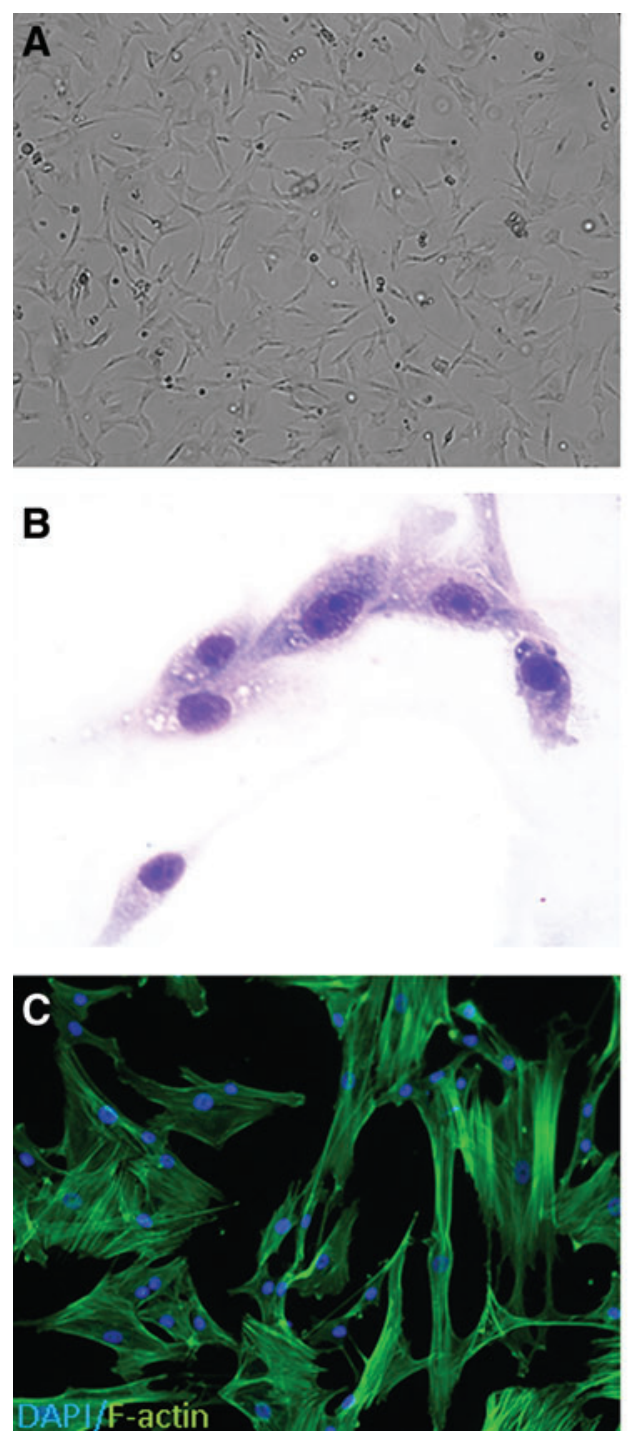
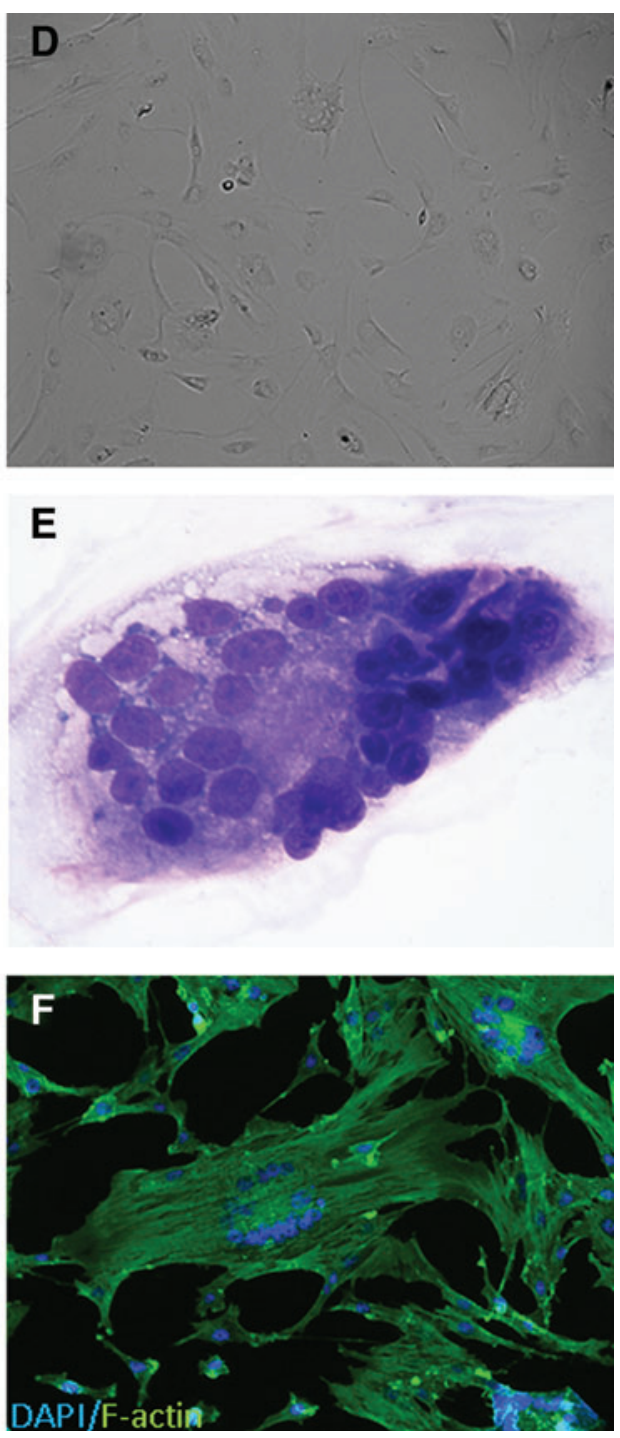

FIG. 2. Syncytial cell formation in fMSCs derived from non-SPF cats. Cellular morphology of normal fMSCs (AC) compared with syncytial cells (D-F). Bright contrast images (A, D 10× objective), Diff-Quik stained images (B, E 40× objective), and fluorescent images $(\mathbf{C}, \mathbf{F} 40 \times$ objective) depict the profound cytopathic effect that is noted in affected cell lines. These changes include marked cellular vacuolation, the formation of giant multinucleated cells. SPF, specific pathogen free. Color images available online at www.liebertpub.com/ scd prevalence of this infection in cat populations [25,26]. FFV can establish latent infection in the host and then induce syncytial cell formation and apoptosis in cultured cells $[27,28]$. Moreover, previous reports have detailed similar effects of simian foamy virus infection in monkey MSCs [29].

The gDNA was extracted from two affected fMSC lines and from peripheral blood mononuclear cells and MSCs derived from an SPF cat for PCR. Both affected fMSC lines had evidence of FFV genomic integration while no PCR product was evident in the samples from the SPF cat (Fig. 3A). The sequence of the PCR amplicons indicated that these two sequences were of FFV origin and that these sequences were almost identical to each other, and were highly homologous with other FFV sequences available in the GenBank database (FFV UCD1 and FFV UCD2; Fig. 3B, C).

To confirm active replication of FFV in fMSCs, we sought to visualize viral particles through transmission electron microscopy (TEM). Upon TEM examination of cultured cells, round particles (ie, putative viral particles) were noted primarily within the cell cytoplasm as well as extracellularly (Fig. 3D). Putative viral particles measured $\sim 100 \mathrm{~nm}$ in diameter with a $\sim 50 \mathrm{~nm}$, round, electron-dense core, a morphology consistent with FFV virions (Fig. 3D). Viral particles, budding from the plasma membrane, were also noted.

We next wanted to investigate whether FFV seropositivity could serve as a screening test to identify cats that will generate FFV-infected MSC cultures. Serum from donors of FFV-positive and FFV-free MSCs were used to probe FFVpositive and FFV-free MSC lysate (Fig. 4). We found that unique bands, consistent with Gag protein p48 and p50, were noted when serum from a donor of FFV-positive MSC culture was used to probe FFV-infected MSC lysate [25]. These bands were absent when the same serum was used to probe FFV-free MSC lysate. These bands were further absent when serum from a donor of an FFV-free MSC culture was used to probe both FFV-positive and FFV-free MSC lysate. These results indicate the presence of FFV-specific IgG antibodies in the serum of a donor of FFV-positive MSCs and the absence of these antibodies in the serum of a donor of FFV-free MSCs.

\section{FFV infection induces syncytial cell formation of fMSCS}

In a further attempt to comply with Koch's postulates [30], we wanted to confirm that exposure of a FFV-free 

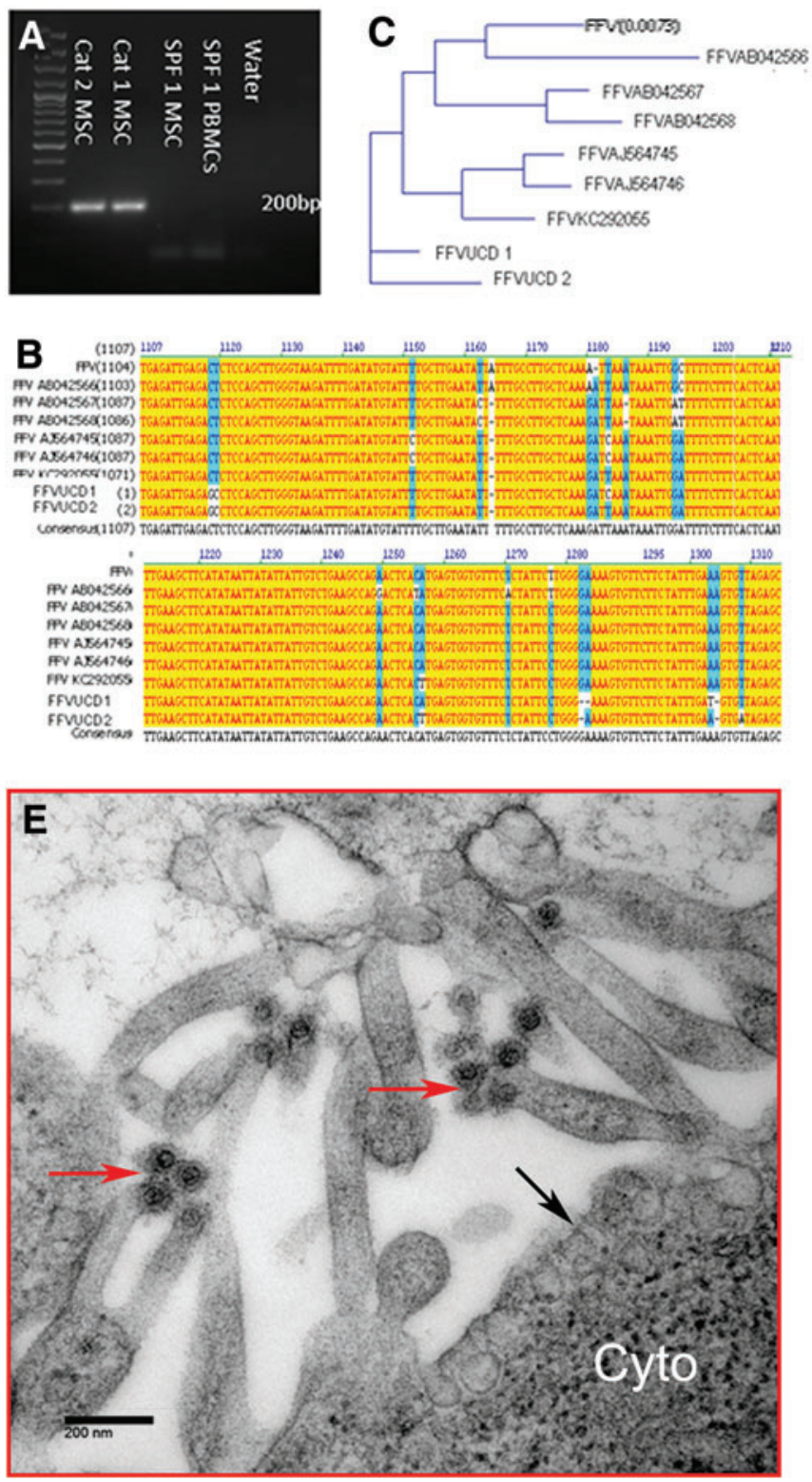

fMSC line to the candidate infectious agent would induce the cytopathic effects (syncytial giant cell formation and proliferation arrest) and that the infectious agent could then be re-isolated from the "diseased" cells. While the control cells maintained typical fMSC morphology throughout the experiment (Fig. 5A), as soon as $48 \mathrm{~h}$ after inoculation, all inoculated cultures contained many syncytial cells (Fig. 5B). To confirm genomic integration and viral gene transcription, gDNA and RNA were extracted from fMSC lines before and after exposure to supernatant. RNA was reverse transcribed to cDNA and PCR was performed to amplify a segment of the FFV genome (Fig. 5C). While the control culture had no evidence (morphologic or molecular) of viral infection, all cultures that were treated with supernatant from infected cell lines had appropriately sized amplicons in both the gDNA and cDNA samples (RT - controls were run to exclude the possibility of vDNA contamination of cDNA),

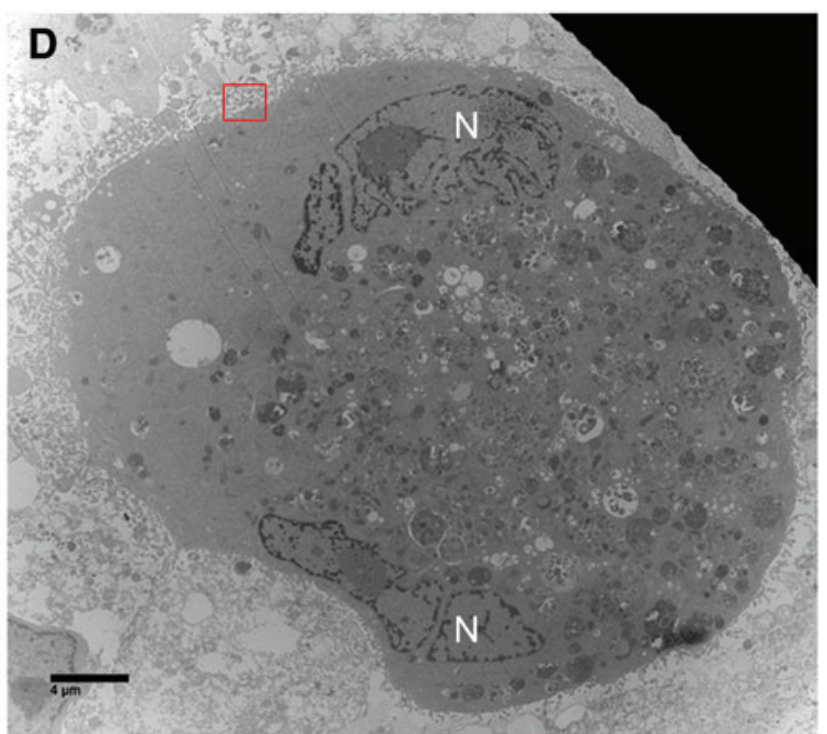

FIG. 3. Cell lines demonstrating cytopathic effect are infected with FFV. The gDNA was extracted from affected MSC lines, SFP cat-derived MSCs and SPF cat-derived peripheral blood mononuclear cells. Standard PCR yielded $\sim 200 \mathrm{bp}$ amplicons, confirming the presence of FFV sequences in the affected cell line and its absence from the SPF cat-derived MSCs and peripheral blood mononuclear cells (A). LTR gene sequence alignment with published FFV sequences (B) confirmed that the amplicons are of FFV origin. Moreover, a dendrogram was created, which indicated that all FFV sequences share high degree of homology (C). Further investigation of affected cell lines through transmission electron microscopy demonstrated the presence of viral particles within multinucleated giant cells (D) The red rectangular is shown in higher magnification in (E). Viral particles, with a morphology that is consistent with FFV, are being shed to the extracellular space from membranal finger-like projections $(\mathbf{E})$. The latter data suggest that virions are being assembled within the cells and are released into the surrounding media. N, nucleus; Cyto, cytoplasm, black arrow, plasma membrane; red arrows, viral particles. gDNA, genomic DNA; PCR, polymerase chain reaction; LTR, long terminal repeat. Color images available online at www.liebertpub.com/scd

indicating the presence of proviral DNA and the transcription of viral RNA.

\section{The antiretroviral drug, tenofovir, restores the proliferative properties of FFV-infected fMSCS with variable viral clearance}

The expansion of high numbers of fMSCs is important for both clinical stem cell therapy for cats with naturally occurring disease as well as for the use of cats as a large animal preclinical model for human diseases and disorders. For many of these conditions, an autologous cell product may be preferable. To determine whether FFV-infected fMSC lines could be rescued and expanded for autologous therapeutic use, we attempted to block viral infection with the reverse transcriptase inhibitor, tenofovir [31]. To establish whether tenofovir may inhibit proliferation or have 


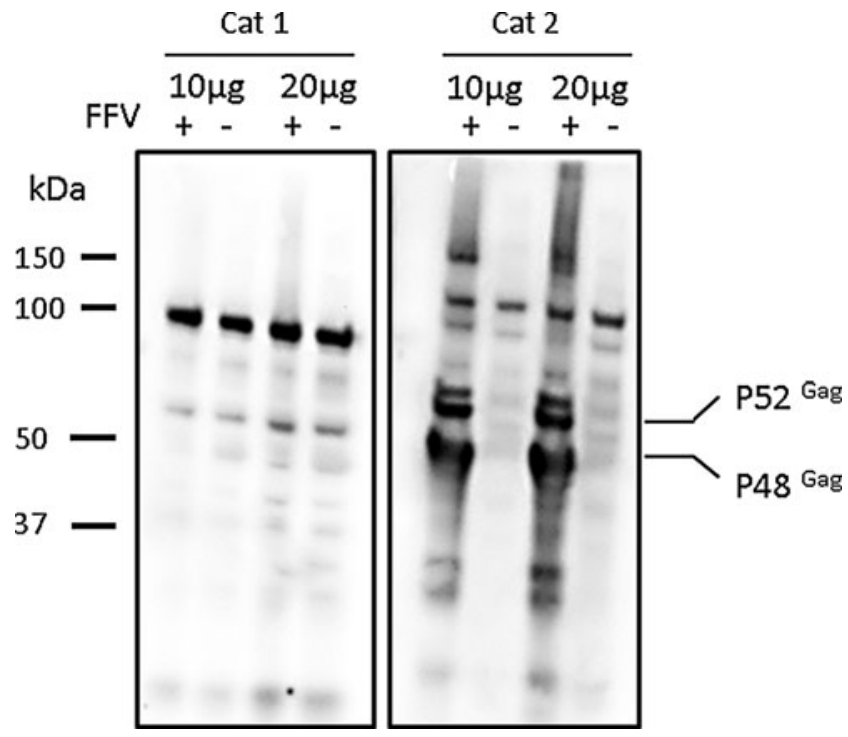

FIG. 4. FFV-specific antibodies could be found in the serum of a donor of FFV-positive MSCs. Serum from cat donors of FFV-free (Cat 1) and FFV-positive (Cat 2) MSCs was used to probe FFV-positive and FFV-free MSC lysates. Unique bands, consistent with FFV Gag protein p52 and $\mathrm{p} 48$, were noted only when serum from a donor of FFVpositive MSCs was used to probe FFV-positive MSC lysate.

cytopathic effects in FFV-free fMSC culture, we determined the growth kinetics and cellular morphology of a FFV-free fMSC line treated with increasing concentrations of tenofovir $(0,1,5,10,50$, and $100 \mu \mathrm{M})$. While lower concentrations $(\leq 10 \mu \mathrm{M})$ did not alter proliferation rate or cellular morphology (Fig. 6A), higher concentrations were associated with proliferation arrest and cell death (data not shown). FFV-infected MSCs were then cultured in culture media with or without noncytopathic concentrations $(1,5$, and $10 \mu \mathrm{M}$ ) of tenofovir, and cell morphology and doubling times were recorded. While FFV-infected fMSC lines that were cultured in complete media (ie, no tenofovir) formed giant multinucleated cells and stopped proliferating at passages 3-5, fMSC lines treated with either 5 or $10 \mu \mathrm{M}$ tenofovir had no morphologic alterations and the proliferative capacity was restored to normal (Fig. 6B, C). PCR analysis for both gDNA and RNA over the course of tenofovir treatment indicated variable viral clearance (data not shown).

\section{Discussion}

We report for the first time that fMSC may be infected with a retrovirus, FFV, which may cause significant cytopathic effects, including proliferation arrest and cell death. We further propose several strategies to facilitate fMSC culture and use, both in a basic science laboratory setting and in a clinical regenerative medicine laboratory.

In our laboratory, we explore regenerative medicine approaches in naturally occurring diseases in large animal species that may be utilized as a model for human disease. Naturally occurring diseases in cats are being increasingly recognized as valid models for human disease that offer various advantages over traditional laboratory animal models [32-38]. To this end, we are exploring FCGS as a candidate animal model of a variety of human immune-mediated disorders with an oral manifestation (ie, lichen planus, Crohn's disease, graft vs. host disease, and pemphigus vulgaris). For these diseases, MSCs have a strong potential to ameliorate inflammation, modulate the immune system, and promote epithelial regeneration.

The fMSC lines we worked with in this study have the typical MSC morphology and cell surface phenotype as broadly described previously for humans, rodents, dogs, horses, and cats
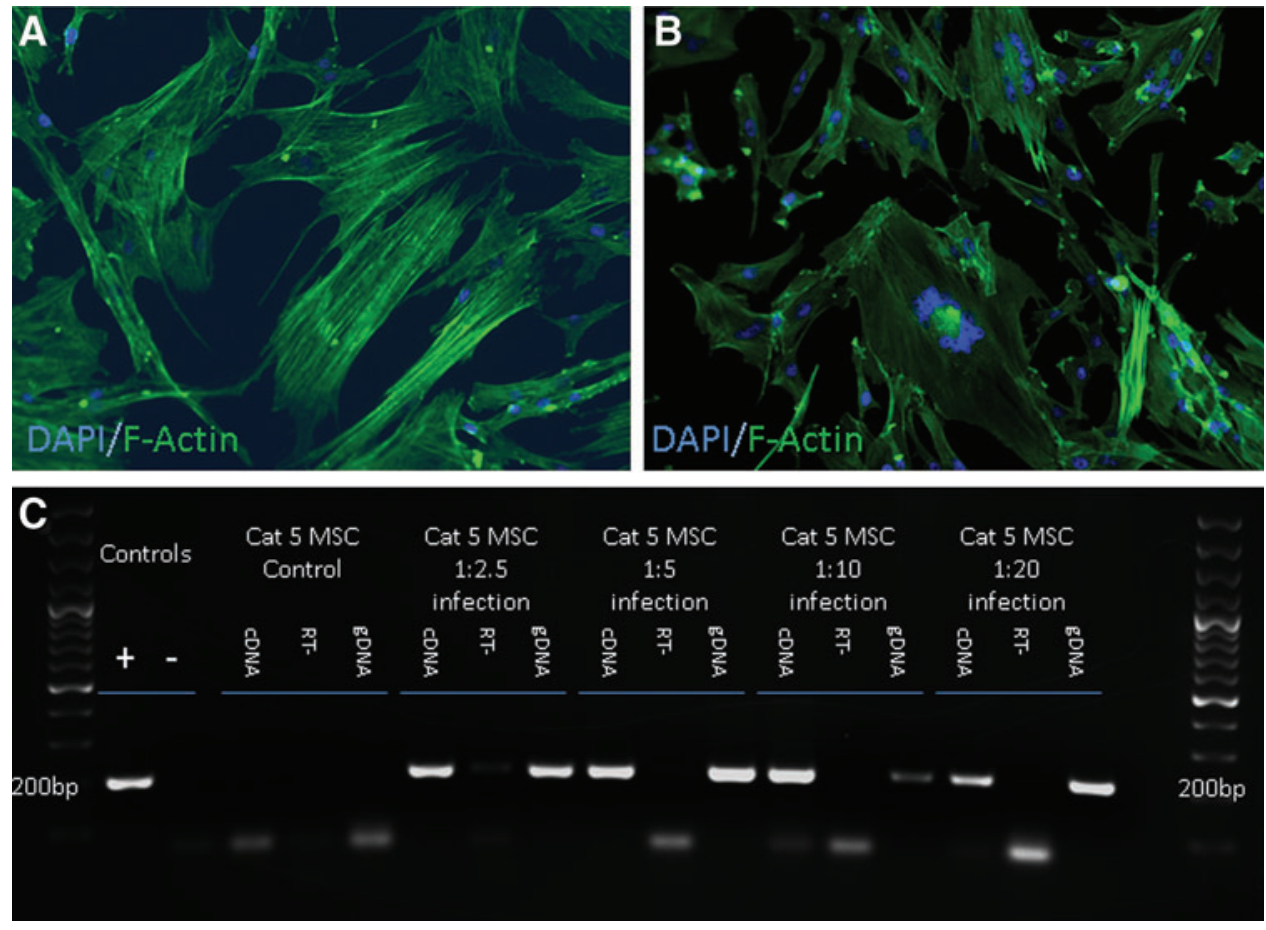

FIG. 5. FFV infection induces syncytial cell formation of fMSCs. FFV-negative cultures were inoculated with various dilutions of cell-free media, obtained from affected cultures. While syncytial cells were never noted in the control cultures (A), multinucleated cells were noted in all inoculated cultures as soon as $48 \mathrm{~h}$ after inoculation (B). Standard PCR (C) further confirmed that control culture was FFV free while all infected cultures had evidence of both viral reverse transcription (ie, positive $206 \mathrm{bp}$ band in the gDNA lanes) as well as evidence of viral genes transcription (positive $206 \mathrm{bp}$ band in the cDNA lanes). The cDNA was confirmed to be free of viral-DNA with the appropriate reverse transcriptase negative control. Color images available online at www.liebertpub.com/scd 

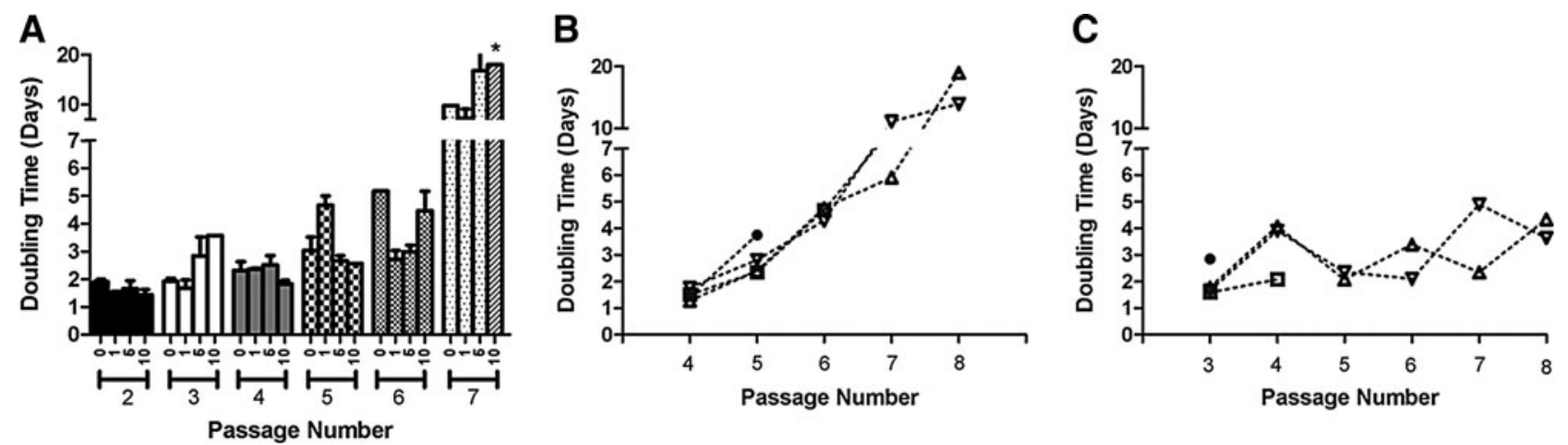

FIG. 6. Tenofovir is safe for use in fMSC culture and may prevent FFV-induced alterations in cell morphology and proliferation. fMSCs from a FFV-free cell line $(n=1)$ were cultured in duplicates with escalating concentrations of tenofovir $(0,1,5,10,50$, and $100 \mu \mathrm{M})$. Cellular doubling times and viability were determined. While vast cell death was noted when tenofovir was used at 50 and $100 \mu \mathrm{M}$, no morphologic or proliferative alterations were noted with concentrations $\leq 10 \mu \mathrm{M}$ (A). Tenofovir was then applied at $0,1,5$, and $10 \mu \mathrm{M}$, in duplicates, to two fMSC lines that were confirmed to be infected with FFV and doubling times were determined $(\mathbf{B}, \mathbf{C})$. While cells that were not treated with tenofovir $(\bullet)$ have stopped proliferating at passage $5(\mathbf{B})$ or $3(\mathbf{C})$, cells that were treated with both $5 \mu \mathrm{M}(\boldsymbol{\Delta})$ and $10 \mu \mathrm{M}(\boldsymbol{\nabla})$ have regained normal proliferative capacity. A $1 \mu \mathrm{M}(\boldsymbol{\bullet})$ concentration was variably effective. Asterisk indicated doubling time $>20$ days.

$[39,40]$. The fMSC growth kinetics has not been previously described; however, it has been noted that fat-derived fMSCs are easier to culture and expand than fMSCs derived from bone marrow [3]. Irrespective of FFV infection, fMSC growth kinetics appears to be blunted and not optimized using standard media conditions that readily support MSC growth and proliferation in humans and other veterinary species [41-43]. Further optimization of culture conditions for the expansion of fMSCs is needed to assure that clinically relevant cell doses (ie, $2-5 \times 10^{6}$ cells $/ \mathrm{kg}$ ) are easily achievable.

FFV is a member of the retroviridae family and spumaretrovirinae subfamily [44]. It has been reported to be present in $20 \%-80 \%$ of cats and not associated with a clinical disorder $[25,26]$. Our work supports the observation that FFV can be noted in $20 \%-80 \%$ of cat cell lines. In addition, FFV is known to induce syncytial cell formation, inhibit cell growth, and induce apoptosis in cultured cells [28]. A similar infection of simian MSCs with simian foamy virus was previously described in primates [29]. FFV replication is associated with syncytial cell formation, decreased proliferation rate, and finally, cell death [28]. We expand the description of FFV infection to primary fMSC lines and report that FFV infection is the direct cause of the cytopathic effect.

There are several strategies that could be employed to manage this inherent challenge in the culture and expansion of fMSC. Regardless of the strategy, we recommend that all cell cultures will be screened for FFV infection status by PCR before cryopreservation. Our study further suggests that FFV seropositivity may be used as a screening method, although further studies to establish the assay's sensitivity and specificity are warranted before its wide use. Moreover, at present, the wide use of a serologic test is hindered by the absence of a commercially available serologic test.

\section{Use of lower passage cells}

In our clinical Regenerative Medicine Laboratory, MSCs are typically grown and passaged twice before cryopreservation. This approach allows for greater MSC expansion and cell yield, along with greater cellular homogeneity and minimal cellular alteration and differentiation. This approach to fMSC culture has not been routinely successful for cats as typically the effects of FFV infection were readily apparent when MSCs were thawed after cryopreservation for expansion to obtain a clinically relevant cell number [passages (P) 3-5]. Our current clinical practice has shifted such that we cryopreserve fMSCs at P1, with a subsequent thaw for expansion and administration of P2-P3 fMSCs. We have not observed any adverse effects of FFV infection on fMSC cultures at $<\mathrm{P} 3$. Advantages of this strategy are that it permits the use of autologous cells and does not require any intervention, such as antiretroviral drugs. Disadvantages of this approach are that the heterogeneity of fMSCs may be higher at lower passage and that there is less flexibility for long-term culture expansion and, as such, high cell doses may be more challenging to acquire. Finally, although this is an exogenous virus with no known in vivo pathologic consequences, the effects of virus infection on MSC function and the potential for adverse effects associated with the readministration of actively replicating FFV to the recipient animal are currently unknown. Regardless, we recommend that each cat be screened for FFV presence before working with its MSCs and appropriate infection prevention measures of other cell lines be implemented.

\section{Antiretroviral treatment of MSC culture}

The early addition of $5 \mu \mathrm{M}$ of tenofovir to fMSC culture media is effective in inhibiting the deleterious effects of FFV infection in fMSCs. The addition of tenofovir to later cell passages, after morphologic alterations have taken place, was not effective at reverting the cells to a proliferative phenotype. This approach permits the use of an autologous cell source and the use of cells at higher passage (ie, P3-P5), which would allow greater expansion potential and greater culture homogeneity. The addition of tenofovir to feline cells in culture has been determined by us and others [45] to have no cytopathic effects in concentrations $<20 \mu \mathrm{M}$. Tenofovir has also been administered to cats, and no adverse effects were noted when administered at $25 \mathrm{mg} / \mathrm{kg}$ to FIV-infected 
cats [46]. Moreover, tenofovir treatment $(10 \mu \mathrm{M})$ did not alter the proliferative capacity, cell surface phenotype, or trilineage differentiation capacity of simian foamy virus-infected monkey MSCs [29]. The disadvantages of this approach are the costs of tenofovir treatment, the lack of consistent viral clearing with the dose we used, and the unknown effects of FFV on fMSC functions and on the recipient host.

\section{Allogeneic use of prescreened $\mathrm{MSC}$ lines}

Cats that are housed in colonies under SPF conditions may potentially have a lower prevalence of FFV infection. As such, fMSC lines from selected FFV-free SPF donors could be established. Moreover, these cell lines could be fully functionally characterized before clinical use, which would overcome natural biologic variation between cell donors. MSC do not express MHC II molecules on their cell surface and have low antigenicity overall $[5,6]$. Although it is still not fully clear if the efficacy and life span of allogeneic MSCs is the same as for autologous MSCs, the use of allogeneic MSCs is widespread and has been shown to be safe in human patients, horses, cats, and others [47-52]. MSCs provoke minimal antigenic stimulation, although allogeneic MSCs have been reported to activate complement in vivo, and thus reducing their half-life time and potentially their effectiveness in the recipient [53].

In conclusion, this is the first study to report the deleterious effects of FFV infection on fMSCs in standard culture conditions. We have determined that fMSC lines that contained abundant syncytial cells were infected with FFV. We have further demonstrated that the phenotypic alterations could be recapitulated in naive cell cultures that were inoculated with supernatant from affected cultures. Importantly, we show molecular evidence of genomic integration and RNA transcription of viral genes in transduced cells, thus complying with Koch's postulates for the determination of causality. We further propose several strategies to manage this problem and discuss advantages and disadvantages of these strategies.

\section{Acknowledgments}

Financial support for this study was provided by the WINN Feline Foundation for a Miller Trust grant and by the George and Phyllis Miller Feline Health Trust of the San Francisco Foundation and administered by the Center of Companion Animal Health, UCD. We thank Scott S. Fish at the California Animal Health and Food Safety Laboratory, School of Veterinary Medicine, UCD, for performing the TEM. The authors also thank Dr. Joe DeRisi and Dr. Jeremy Horst from the University of California, San Francisco, for their valuable consultation. Finally, we also thank Megan Badgley and Erica Becerra for their instrumental support and assistance.

\section{Author Disclosure Statement}

No competing financial interests exist.

\section{References}

1. de Mattos CA, AL Alves, MA Golim, A Moroz, CA Hussni, PG de Oliveira and E Deffune. (2009). Isolation and immunophenotypic characterization of mesenchymal stem cells derived from equine species adipose tissue. Vet Immunol Immunopathol 132:303-306.

2. Guercio A, S Di Bella, S Casella, P Di Marco, C Russo and G Piccione. (2013). Canine mesenchymal stem cells (MSCs): characterization in relation to donor age and adipose tissueharvesting site. Cell Biol Int 37:789-798.

3. Webb TL, JM Quimby and SW Dow. (2012). In vitro comparison of feline bone marrow-derived and adipose tissue-derived mesenchymal stem cells. J Feline Med Surg 14:165-168.

4. Zuk PA, M Zhu, H Mizuno, J Huang, JW Futrell, AJ Katz, P Benhaim, HP Lorenz and MH Hedrick. (2001). Multilineage cells from human adipose tissue: implications for cell-based therapies. Tissue Eng 7:211-228.

5. Carrade DD and DL Borjesson. (2013). Immunomodulation by mesenchymal stem cells in veterinary species. Comp Med 63:207-217.

6. Peroni JF and DL Borjesson. (2011). Anti-inflammatory and immunomodulatory activities of stem cells. Vet Clin North Am Equine Pract 27:351-362.

7. Singer NG and AI Caplan. (2011). Mesenchymal stem cells: mechanisms of inflammation. Annu Rev Pathol 6:457-478.

8. Carrade DD, MW Lame, MS Kent, KC Clark, NJ Walker and DL Borjesson. (2012). Comparative analysis of the immunomodulatory properties of equine adult-derived mesenchymal stem cells. Cell Med 4:1-11.

9. Tolar J, K Le Blanc, A Keating and BR Blazar. (2010). Concise review: hitting the right spot with mesenchymal stromal cells. Stem Cells 28:1446-1455.

10. Wood JA, I Ly, DL Borjesson, PF Nealey, P Russell and CJ Murphy. (2012). The modulation of canine mesenchymal stem cells by nano-topographic cues. Exp Cell Res 318:24382445.

11. Hartman KG, JD Bortner, GW Falk, J Yu, MG Martin, AK Rustgi and JP Lynch. (2013). Modeling inflammation and oxidative stress in gastrointestinal disease development using novel organotypic culture systems. Stem Cell Res Ther 4 Suppl 1:S5.

12. Herrmann RP and MJ Sturm. (2014). Adult human mesenchymal stromal cells and the treatment of graft versus host disease. Stem Cells Cloning 7:45-52.

13. Martinez-Montiel MP, GJ Gomez-Gomez and AI Flores. (2014). Therapy with stem cells in inflammatory bowel disease. World J Gastroenterol 20:1211-1227.

14. Ricart E, A Jauregui-Amezaga, I Ordas, S Pino, AM Ramirez and J Panes. (2013). Cell therapies for IBD: what works? Curr Drug Targets 14:1453-1459.

15. Arzi B, ER Wisner, DJ Huey, PH Kass, J Hu and KA Athanasiou. (2012). A proposed model of naturally occurring osteoarthritis in the domestic rabbit. Lab Anim (NY) 41:20-25.

16. McAllister MM. (2014). Successful vaccines for naturally occurring protozoal diseases of animals should guide human vaccine research. A review of protozoal vaccines and their designs. Parasitology 141:624-640.

17. Simpson RM, BC Bastian, HT Michael, JD Webster, ML Prasad, CM Conway, VM Prieto, JM Gary, MH Goldschmidt, et al. (2014). Sporadic naturally occurring melanoma in dogs as a preclinical model for human melanoma. Pigment Cell Melanoma Res 27:37-47.

18. Arzi B, B Murphy, DP Cox, N Vapniarsky, PH Kass and FJ Verstraete. (2010). Presence and quantification of mast cells in the gingiva of cats with tooth resorption, periodontitis and chronic stomatitis. Arch Oral Biol 55:148-154. 
19. Harley R, TJ Gruffydd-Jones and MJ Day. (2011). Immunohistochemical characterization of oral mucosal lesions in cats with chronic gingivostomatitis. J Comp Pathol 144:239-250.

20. Hennet PR, GA Camy, DM McGahie and MV Albouy. (2011). Comparative efficacy of a recombinant feline interferon omega in refractory cases of calicivirus-positive cats with caudal stomatitis: a randomised, multi-centre, controlled, double-blind study in 39 cats. J Feline Med Surg 13:577-587.

21. Chung DJ, K Hayashi, CA Toupadakis, A Wong and CE Yellowley. (2012). Osteogenic proliferation and differentiation of canine bone marrow and adipose tissue derived mesenchymal stromal cells and the influence of hypoxia. Res Vet Sci 92:66-75.

22. Kol A, S Foutouhi, NJ Walker, N Kong, BC Weimer and DL Borjesson. (2014). Gastrointestinal microbes interact with canine adipose derived mesenchymal stem cells in vitro and enhance immunomodulatory functions. Stem Cells Dev 23:1831-1843.

23. Wood JA, DJ Chung, SA Park, AL Zwingenberger, CM Reilly, I Ly, NJ Walker, W Vernau, K Hayashi, et al. (2012). Periocular and intra-articular injection of canine adipose-derived mesenchymal stem cells: an in vivo imaging and migration study. J Ocul Pharmacol Ther 28: 307-317.

24. Vidal MA, GE Kilroy, JR Johnson, MJ Lopez, RM Moore and JM Gimble. (2006). Cell growth characteristics and differentiation frequency of adherent equine bone marrowderived mesenchymal stromal cells: adipogenic and osteogenic capacity. Vet Surg 35:601-610.

25. Bleiholder A, M Muhle, T Hechler, S Bevins, S Vandewoude, J Denner and M Lochelt. (2011). Pattern of seroreactivity against feline foamy virus proteins in domestic cats from Germany. Vet Immunol Immunopathol 143: 292-300.

26. Winkler IG, M Lochelt and RL Flower. (1999). Epidemiology of feline foamy virus and feline immunodeficiency virus infections in domestic and feral cats: a seroepidemiological study. J Clin Microbiol 37:2848-2851.

27. Hatama S, K Otake, M Ohta, M Kobayashi, K Imakawa, A Ikemoto, H Okuyama, M Mochizuki, T Miyazawa, et al. (2001). Reactivation of feline foamy virus from a chronically infected feline renal cell line by trichostatin A. Virology 283:315-323.

28. Ikeda Y, S Itagaki, S Tsutsui, Y Inoshima, M Fukasawa, K Tomonaga, Y Tohya, K Maeda, M Mochizuki, T Miyazawa and T Mikami. (1997). Replication of feline syncytial virus in feline T-lymphoblastoid cells and induction of apoptosis in the cells. Microbiol Immunol 41:431-435.

29. Lee CC, F Ye and AF Tarantal. (2006). Comparison of growth and differentiation of fetal and adult rhesus monkey mesenchymal stem cells. Stem Cells Dev 15:209-220.

30. Inglis TJ. (2007). Principia aetiologica: taking causality beyond Koch's postulates. J Med Microbiol 56:1419-1422.

31. De Clercq E. (2005). Antiviral drug discovery and development: where chemistry meets with biomedicine. Antiviral Res 67:56-75.

32. Aznavour N, C Cendres-Bozzi, L Lemoine, C Buda, JP Sastre, Z Mincheva, L Zimmer and JS Lin. (2012). MPTP animal model of Parkinsonism: dopamine cell death or only tyrosine hydroxylase impairment? A study using PET imaging, autoradiography, and immunohistochemistry in the cat. CNS Neurosci Ther 18:934-941.
33. Bosshard NU, M Hubler, S Arnold, J Briner, MA Spycher, HJ Sommerlade, FK von and R Gitzelmann. (1996). Spontaneous mucolipidosis in a cat: an animal model of human I-cell disease. Vet Pathol 33:1-13.

34. Fox PR, BJ Maron, C Basso, SK Liu and G Thiene. (2000). Spontaneously occurring arrhythmogenic right ventricular cardiomyopathy in the domestic cat: a new animal model similar to the human disease. Circulation 102:1863-1870.

35. Green ST and FA Green. (1987). The Manx cat: an animal model for neural tube defects. Mater Med Pol 19:219-221.

36. Kolahian S, AA Shahbazfar, H Tayefi-Nasrabadi, R Keyhanmanesh, K Ansarin, H Ghasemi, AH Rashidi, R Gosens and M Hanifeh. (2014). Tiotropium effects on airway inflammatory events in the cat as an animal model for acute cigarette smoke-induced lung inflammation. Exp Lung Res 40:272-287.

37. Narfstrom K, DK Holland and M Menotti-Raymond. (2011). The domestic cat as a large animal model for characterization of disease and therapeutic intervention in hereditary retinal blindness. J Ophthalmol 2011:906943.

38. Willett BJ, JN Flynn and MJ Hosie. (1997). FIV infection of the domestic cat: an animal model for AIDS. Immunol Today 18:182-189.

39. Munoz JL, SJ Greco, SA Patel, LS Sherman, S Bhatt, RS Bhatt, JA Shrensel, YZ Guan, G Xie, et al. (2012). Feline bone marrow-derived mesenchymal stromal cells (MSCs) show similar phenotype and functions with regards to neuronal differentiation as human MSCs. Differentiation 84:214-222.

40. Quimby JM, TL Webb, DS Gibbons and SW Dow. (2011). Evaluation of intrarenal mesenchymal stem cell injection for treatment of chronic kidney disease in cats: a pilot study. J Feline Med Surg 13:418-426.

41. Bruder SP, N Jaiswal and SE Haynesworth. (1997). Growth kinetics, self-renewal, and the osteogenic potential of purified human mesenchymal stem cells during extensive subcultivation and following cryopreservation. J Cell Biochem 64:278-294.

42. Tawonsawatruk T, A Spadaccino, IR Murray, B Peault and HA Simpson. (2012). Growth kinetics of rat mesenchymal stem cells from 3 potential sources: bone marrow, periosteum and adipose tissue. J Med Assoc Thai 95 Suppl 10:S189-S197.

43. Vidal MA, NJ Walker, E Napoli and DL Borjesson. (2012). Evaluation of senescence in mesenchymal stem cells isolated from equine bone marrow, adipose tissue, and umbilical cord tissue. Stem Cells Dev 21:273-283.

44. Rethwilm A and J Bodem. (2013). Evolution of foamy viruses: the most ancient of all retroviruses. Viruses 5: 2349-2374.

45. Greggs WM, III, CL Clouser, SE Patterson and LM Mansky. (2012). Discovery of drugs that possess activity against feline leukemia virus. J Gen Virol 93:900-905.

46. Vahlenkamp TW, A De Ronde, J Balzarini, L Naesens, E De Clercq, MJ van Eijk, MC Horzinek and HF Egberink. (1995). (R)-9-(2-phosphonylmethoxypropyl)-2,6-diaminopurine is a potent inhibitor of feline immunodeficiency virus infection. Antimicrob Agents Chemother 39:746-749.

47. Carrade DD, SD Owens, LD Galuppo, MA Vidal, GL Ferraro, F Librach, S Buerchler, MS Friedman, NJ Walker and DL Borjesson. (2011). Clinicopathologic findings following intra-articular injection of autologous and allogeneic placentally derived equine mesenchymal stem cells in horses. Cytotherapy 13:419-430. 
48. Carrade DD, VK Affolter, CA Outerbridge, JL Watson, LD Galuppo, S Buerchler, V Kumar, NJ Walker and DL Borjesson. (2011). Intradermal injections of equine allogeneic umbilical cord-derived mesenchymal stem cells are well tolerated and do not elicit immediate or delayed hypersensitivity reactions. Cytotherapy 13:1180-1192.

49. Lalu MM, L McIntyre, C Pugliese, D Fergusson, BW Winston, JC Marshall, J Granton and DJ Stewart. (2012). Safety of cell therapy with mesenchymal stromal cells (SafeCell): a systematic review and meta-analysis of clinical trials. PLoS One 7:e47559.

50. Poh KK, E Sperry, RG Young, T Freyman, KG Barringhaus and CA Thompson. (2007). Repeated direct endomyocardial transplantation of allogeneic mesenchymal stem cells: safety of a high dose, "off-the-shelf", cellular cardiomyoplasty strategy. Int J Cardiol 117:360-364.

51. Prasad VK, KG Lucas, GI Kleiner, JA Talano, D Jacobsohn, G Broadwater, R Monroy and J Kurtzberg. (2011). Efficacy and safety of ex vivo cultured adult human mesenchymal stem cells (Prochymal) in pediatric patients with severe refractory acute graft-versus-host disease in a compassionate use study. Biol Blood Marrow Transplant 17:534-541.
52. Quimby JM, TL Webb, LM Habenicht and SW Dow. (2013). Safety and efficacy of intravenous infusion of allogeneic cryopreserved mesenchymal stem cells for treatment of chronic kidney disease in cats: results of three sequential pilot studies. Stem Cell Res Ther 4:48.

53. Li Y and F Lin. (2012). Mesenchymal stem cells are injured by complement after their contact with serum. Blood 120:3436-3443.

Address correspondence to: Dr. Boaz Arzi

Department of Surgical and Radiological Sciences School of Veterinary Medicine University of California, Davis One Shield Avenue Davis, CA 95616

E-mail: barzi@ucdavis.edu

Received for publication July 1, 2014 Accepted after revision November 17, 2014 Prepublished on Liebert Instant Online November 17, 2014 